\title{
Effect of different drying methods on chemical and molecular structure of heteropolysaccharide-protein gum from durian seed.
}

\begin{abstract}
The functional properties and biological aspects of a natural biodegradable biopolymer depend on its chemical and molecular structure. In this study, the effect of different drying processes on the chemical and molecular structure of the natural biodegradable biopolymer from durian seed was investigated. The chemical structure was analyzed by assessing the carbohydrate profile, protein, amino acid composition, moisture, and ash. Molecular weight $(\mathrm{Mw})$, number average molecular weight $(\mathrm{Mn}), \mathrm{Mw} / \mathrm{Mn}$ ratio and mass recovery were assessed by using a size-exclusion chromatography coupled to multi angle laser lightscattering (SEC-MALS). The present study revealed that main monosaccharides in the chemical structure of differently dried durian seed gums were galactose (50.1-64.9\%), glucose $(29.4-45.7 \% \%)$, arabinose $(0.11-0.89 \%)$, and xylose $(0.019-0.86 \%)$. The protein analysis indicated the presence of a low amount of the protein fraction (3.2-3.9\%) in the chemical structure of the biopolymer from durian seed. The most abundant amino acids in the chemical structure of durian seed gum were leucine (31.78-43.02\%), lysine (6.23-7.78\%), aspartic acid (6.45-8.58\%), glycine (6.17-7.27\%), glutamic acid (5.43-6.55\%), alanine $(4.60-6.23 \%)$, and valine (4.49-5.52). The current study exhibited that the biodegradable biopolymer from durian seed was a heteropolysaccharideprotein complex with medium $\mathrm{Mw}$ ranging from $1.06 \times 105$ to $1.15 \times 105(\mathrm{~g} / \mathrm{mol})$.
\end{abstract}

Keyword: Biodegradable biopolymer; Chemical structure; Molecular weight; Sugar composition; Amino acid composition. 